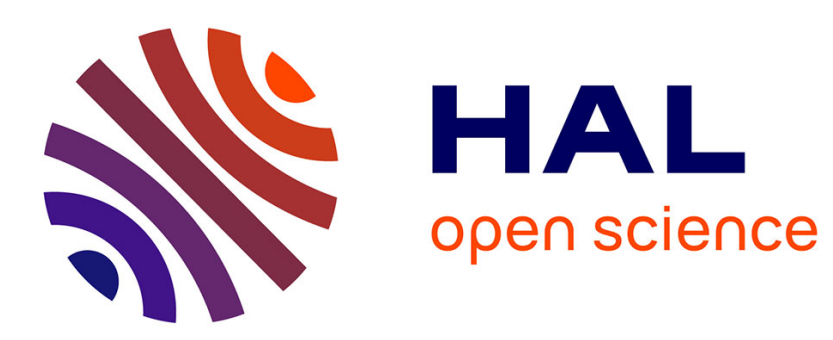

\title{
THE SPECTROSCOPY OF SURFACE PHONONS BY INELASTIC ATOM SCATTERING
}

\author{
G. Benedek, G. Brusdeylins, R. Doak, J. Toennies
}

\section{To cite this version:}

G. Benedek, G. Brusdeylins, R. Doak, J. Toennies. THE SPECTROSCOPY OF SURFACE PHONONS BY INELASTIC ATOM SCATTERING. Journal de Physique Colloques, 1981, 42 (C6), pp.C6-793-C6-800. 10.1051/jphyscol:19816234 • jpa-00221319

\section{HAL Id: jpa-00221319 https://hal.science/jpa-00221319}

Submitted on 1 Jan 1981

HAL is a multi-disciplinary open access archive for the deposit and dissemination of scientific research documents, whether they are published or not. The documents may come from teaching and research institutions in France or abroad, or from public or private research centers.
L'archive ouverte pluridisciplinaire HAL, est destinée au dépôt et à la diffusion de documents scientifiques de niveau recherche, publiés ou non, émanant des établissements d'enseignement et de recherche français ou étrangers, des laboratoires publics ou privés. 
JOURNAL DE PHYSIQUE

Colloque 66 , supplément au $n^{\circ} 12$, Tome 42 , décembre 1981

page $\quad 66-793$

\title{
THE SPECTROSCOPY OF SURFACE PHONONS BY INELASTIC ATOM SCATTERING
}

\author{
G. Benedek, G. Brusdeylins*, R.B. Doak ${ }^{*}$ and J.P. Toennies* \\ Gruppo Nazionale di Struttura della Materia del Consiglio Nazionale delle \\ Ricerche, Istituto di Fisica dell'Universita, I-20133 Milano, Italy \\ * Max-Planck-Institut für Strömungsforschung, Böttinger Str. 4-8, D-3400 \\ cöttingen, F.R.G.
}

\begin{abstract}
The recent great advance in the production of highly monochromatic atomic beams is opening new perspectives in surface physics, having made way to a full determination of the surface vibrational structure. After a short review of the earlier attempts to detect surface phonons from the angular distributions of scattered atoms, we report on the direct measurement of surface phonon dispersion curves, first achieved by Brusdeylins, Doak and Toennies in alkali halides, from time-of-flight (TOF) spectra of scattered He atoms. A comparison is made with the existing theories of surface phonons in ionic crystals. The state of the art in the theory of inelastic scattering processes is briefly illustrated in order to discuss the theoretical interpretation of TOF spectra. The one-phonon energy-loss spectra of He scattered from Lif(OO1) calculated for a hard corrugated surface model are found to be in good agreement with TOF spectra at all the incidence angles. Evidence is given that, in addition $t_{1}$ ayleigh waves, important contributions to the inelastic scatte $g$ come from the surfaceprojected density of bulk phonons. The possible observation of optical surface modes in $\mathrm{KCl}(\mathrm{OO} 1)$ is finally discussed.
\end{abstract}

1. Introduction.- Although surface phonons have since Iong attracted much attention due to their role in several surface and interface phenomena and in various technological applications, 1 their spectroscopy in the dispersive region has been considered till now much more difficult than that of bulk phonons. Indeed the conventional probes of bulk phonons, such as neutrons ${ }^{2}$ and light, are only weakly sensitive to the surface owing to their large penetration into the solid. In the plasma spectral region photons become surface sensitive but couple only to very long-wave surface excitations. ${ }^{3} 4$ Also electron energy loss spectroscopy, which has given us the first evidence of surface electromagnetic modes in monocrystals, 5 and inelastic electron tunneling spectroscopy 6 are actually restricted to long waves by unfavorable kinematical conditions.

The great potentialities of atom scattering in surface phonon spectroscopy have been apparent since the theoretical work of Cabrera, Celi $i$ and Manson, 7 sut only the recent great advance in the production and detection of highly monochromatic atomic beams, triggered off by the studies in rarified gas dynamics, 8 has made way for a full determination of the surface vibrational structure. Today atoms can do for surface phonons the same job that slow neutrons do for bulk phonons.

Alongside, various theoretical problems had to be considered, since the assessment of such a powerful technique in surface phonon analysis required an accurate comparison with the predicted energy-loss profiles and related surface phonon densities. This in order to answer 
two urgent questions: i) Whether and when are single-phonon processes predominant; $i i)$ in how distorted a way do energy-loss spectra reflect the surface projected phonon density.

2. Inelastic processes in angular distributions.- The early experiments carried out by Subbarao and Miller ${ }^{9}$ with cold He beams on Ag(111) provided a first clear separation between elastic and inelastic scattering, the latter displaying a clear multiphonon nature.

on the contrary, the data obtained by Williams and Mason for He scattering from LiF(OO1) 10 and NaF(OO1) 11 were indicative of one-phonon processes. The phonon frequencies, derived from a sophisticated analysis of the out-of-plane angular distributions (AD) around the diffraction peaks, are in fair agreement with the calculated Rayleigh wave (RW) dispersion curves.

In fig. la the data for $\mathrm{NaF}$ are compared to the theoretical curve obtained by the Green's function method 12 , using the breathing shell model (BSM) and room temperature data. Shell model slab calculations ${ }^{13}$ give almost identical results.

The results of Williams and Mason are quite remarkable when considered in light of the recent data obtained by Doak et $a l^{14}$ by the time-of-flight (TOF) technique and a much better resolution (fig. Ib).
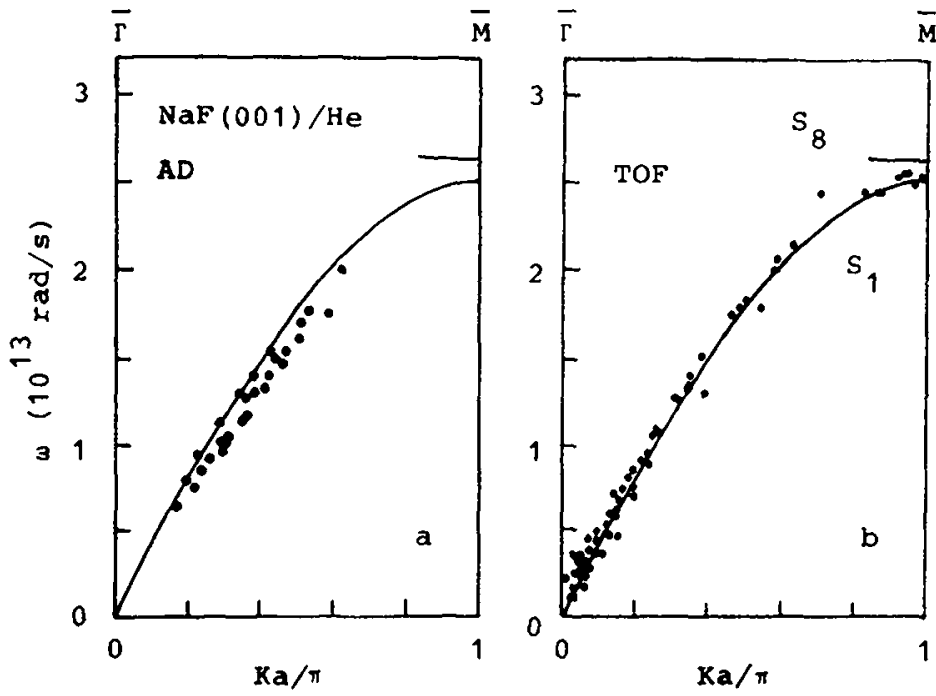

Fig. 1 : Calculated Rayleigh wave dispersion curve in NaF(OOl) compared to $\mathrm{He}^{4}$ scattering data obtained from angular distributions (a: ref.11) and time-of-flight spectra (b: ref. 14).

According to Avila and Lagos 15 , the systematic deviation in fig.la from the thoretical curve is removed when the data are analyzed in terms of kinematical focussing.

The kinematical focussing (KF) occurs with any particular scattering geometry for which the paraboloid representing the energy-loss versus momentum transfer relation 


$$
\left.\omega / \omega_{i}=1-I_{-}\left(1-K_{p} / K_{i}\right)^{2}+K_{n p}^{2} / K_{i-}^{2}\right]\left(\sin \theta_{i} / \sin \theta_{f}\right)^{2}
$$

is tangent to a surface-phonon dispersion surface $\omega=\omega(\vec{K}) .16$ Here $\omega_{i}$ and $K_{i}$ are energy and parallel momentum of the incident atom $(\kappa=1)^{i}$, $\theta_{i}$ and $\theta_{f}$ the incidence and outgoing angles, respectively. $K_{p}$ and $K_{n p}$ are the components of the transferred parallel momentum $\vec{k}=\left(K_{p}, K_{n p}\right)$, respectively parallel and normal to the incidence plane.

$\mathrm{KF}$ yields singularities in the angular distribution due to the vanishing of the Jacobian transforming momentum-space coordinates into angular coordinates.

For planar scattering $\left(K_{n p}=0\right), K F$ may occur only along symmetry directions. For relatively heavy probes, like $\mathrm{Ne}$, and large $\theta_{f}$ and $\theta_{i}$, $\mathrm{KF}$ occurs at small phonon group velocities, i.e. for phonons close to the critical points, where the phonon density is large. ${ }^{16}$ Avila and Lagos have discussed another important enhancement mechanism for non-planar KF which explains Williams and Mason's data. 15

Boato and Cantini 17 performed a careful investigation of the angular distributions of Ne planar scattering from LiF(0O1), finding a rich fine structure in addition to the elastic diffraction pattern. Although interpreting such inelastic features as due to $\mathrm{KF}$ yielded frequencies of surface modes in reasonable agreement with the theoretical prediction for Rayleigh and Lucas modes at $\bar{M}$ and $\bar{\Gamma}$ critical points ${ }^{18}$, Cantini, Felcher and Tatarek found a more convincing explanation of the fine structure in terms of inelastic resonances with surface bound states ${ }^{19}$. In addition, their kinematical analysis led to a rough determination of RW dispersion. Cantini and Tatarek have made a similar analysis for the inelastic resonances of He scattered from graphite (0001).20

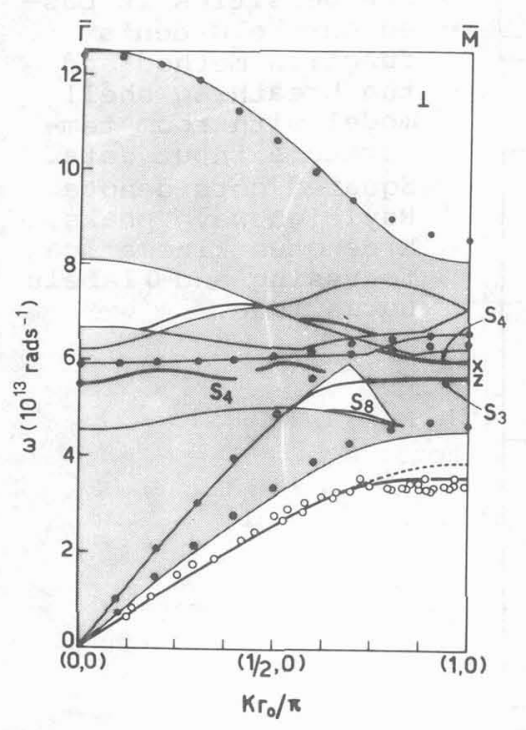

The high-resolution angular distributions recently obtained by Brusdeylins et $a l$ for He scattering from LiF(OOl) and NaF(OO1) 21 give only a faint evidence of KF effect, its features being hardly distinguishable from the complicated pattern of inelastic resonances.

Thus kF could be visible only where the channels to bound states are extremely weak, as in metals, but we do not know of any example, apart from some poorly understood data on He-Au (111). 22

However, in view of the recent advance in the theory and design ${ }^{8}$ of highly monochromatic nozzle beam sources, the KF way to surface phonons has been abandoned in favour of TOF spectroscopy.

Fig. 2 : Surface phonon dispersion curves in LiF(OO1) along (1OO) for sagittal polarization. Comparison is made with He 4 scattering data (0) and neutron data (•). 
3. The analysis of time-of-flight spectra.- In the late seventies ToF measurements provided a direct evidence of Rayleigh waves in the THz range 23.24 . The high-resolution spectra measured by Toennies group in Göttingen led for the first time to the full determination of the RW dispersion as well as of the energy loss profiles in LiF ${ }^{25}$, NaF ( $f i g$. 1b) and $\mathrm{KCl} .{ }^{14}$

These experiments have stimulated a new effort in the theory of inelastic processes 26-30. Most of the features found in ToF spectra-dominance of RWs and cut-off of optical frequencies - were predicted already in the framework of the distorted wave Born approximation (DWBA) 3.1

The validity of this theory for ionic crystals, where the surface is quite corrugated, is in question, however, since the non-specular part of the potential works as a perturbation. Physically it would mean that unklapp processes involving surface-reciprocal lattice vectors $G \neq O$ have to be less probable, which is clearly in contrast with the observation of strong quantum rainbow effects.17
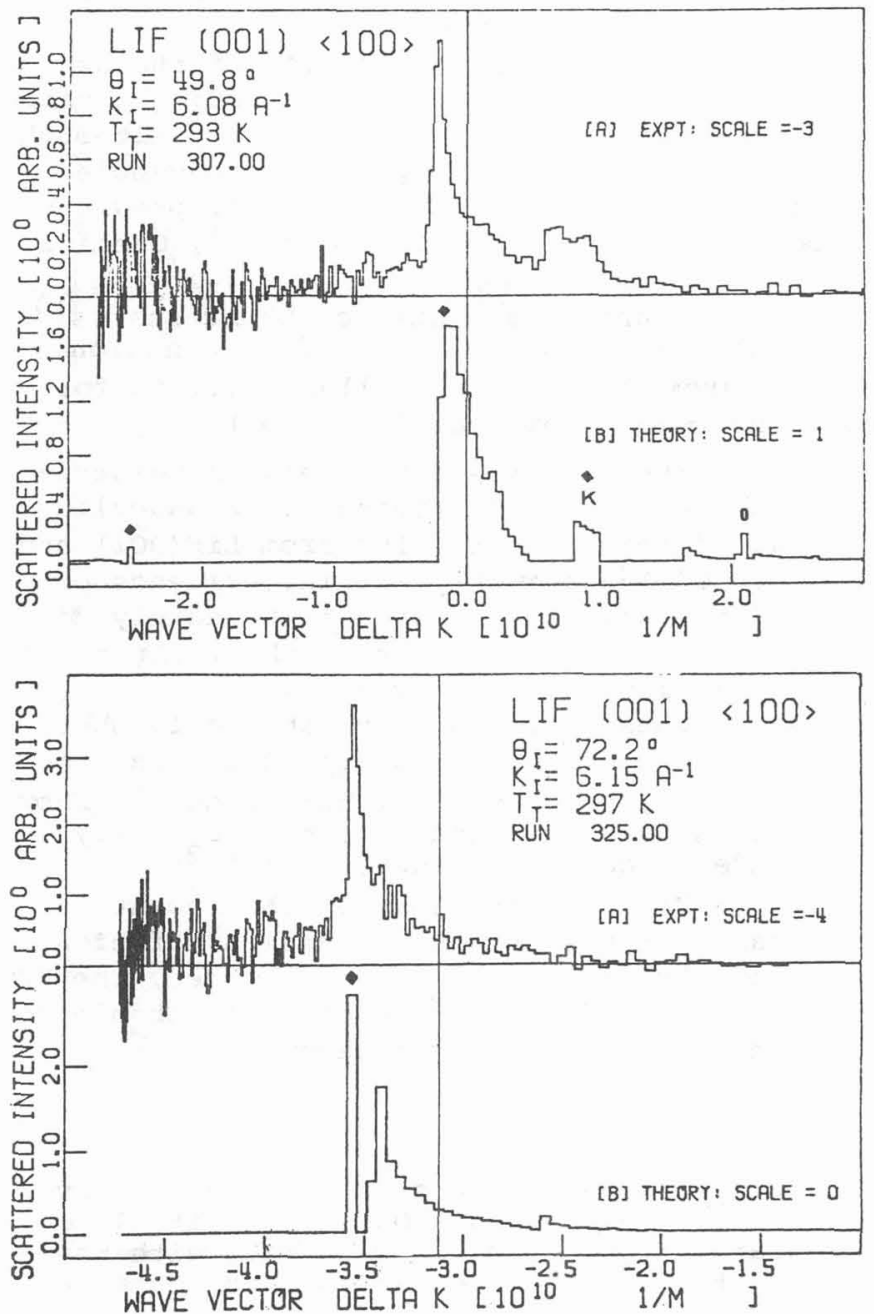

Fig. 3 : Time-offlight spectra of $\mathrm{He}$ scattered from LiF (001) surface along (100) for incidence angle $\theta_{i}=49.8^{\circ}$ and $72.2^{\circ}$ (Erom ref. 14) and calculated onephonon reflection coefficient for a hard corrugated surface model. The calculation of the phonon densities is based on the Green"s function method and the breathing shell model with room temperature input data. Squared dots denote Rayleigh wave peaks, $\mathrm{K}$ denotes kinematical focussing and 0 labels Lucas mode. 


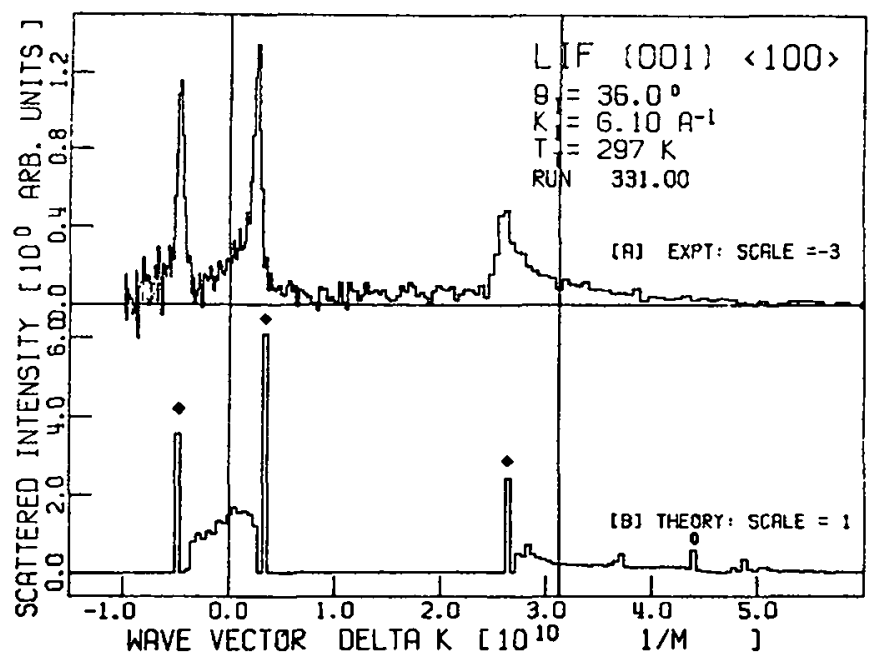

Fig. 4 : Same as fig. 3 for incidence angle $\theta_{i}=36.0^{\circ}$.

Thus in the theoretical interpretation of TOF data our main concern is the non-perturbative treatment of the static corrugated potential. We work in a DWBA where only the phonon-induced corrugation acts as a perturbation. The one-phonon reflection coefficient for a process casting an incident atom of momentum $\vec{k}_{j}$ into a final state $k_{f}$ oriented within a solid angle $d \Omega$, is expressed by ${ }^{28}$

$$
\frac{d^{2} R(1)}{d \omega d \Omega}=\frac{k^{2}}{8 \pi^{3}}\left|\frac{k_{f}}{k_{i z}}\right|\left|1+\operatorname{coth} \frac{\omega}{2 k_{B} T}\right| \sum_{K K^{\prime} \alpha \beta} g_{K \alpha}(\vec{K}) g_{K^{\prime} B}^{*}(\vec{K}) \rho_{K \alpha, K^{\prime} B}(\vec{K}, \omega),
$$
where $T$ is the surface temperature, and $\rho_{k \alpha, k^{\prime},}(\vec{K}, \omega)$ are the elements
of surface-projected phonon density matrix.

If $\mathbf{z}=D_{O}(\vec{R})$ represents the static surface profile, $D_{O}(\vec{R})$ being a periodic function of the position vector $\vec{R}=(x, y)$, the coupling coefficients are given by

$$
g_{k \alpha}(\vec{K})=\int d \vec{R} \exp \left\{-W(\vec{R})-i \vec{K} \cdot \vec{R}+i k_{z} D_{0}(\vec{R})\right\} g_{0}(\vec{R}) \partial D_{0}(\vec{R}) / \partial u_{k \alpha^{\prime}}
$$

where $\partial D_{0}(\vec{R}) / \partial u_{k \alpha}$ is the distortion of the surface profile due to a unit displacement $\vec{u}_{k}$ of the $k-t h$ ion, and $w(\vec{R})$ is the Debye-Waller factor. The scattering amplitude $g_{0}(\vec{R})$ is obtained by solving numerically the Lippmann-Schwinger equation in the direct space for the static corrugated surface, using a method developed by Garcia and Cabrera..$^{32}$

The surface-projected phonon densities are calculated by the Green's function method, 12 for 33 values of $\overrightarrow{\mathrm{K}}$ in the irreducible segment $\overline{\Gamma M}$ lying in the scattering plane, and for 100 equally-spaced values of $\omega$ between $O$ and the maximum crystal frequency. This calculation is equivalent to a slab calculation with 192 layers.

Fig. 2 shows the dispersion curves along (100) of Lif(0O1) surface modes with sagittal polarization - the one involved in planar scattering.Heavy lines are surface modes. Thin lines are band edges of bulk modes having non-vanishing sagittal component. When a dispersion curve enters a band, the surface-localized mode transforms into a reso- 


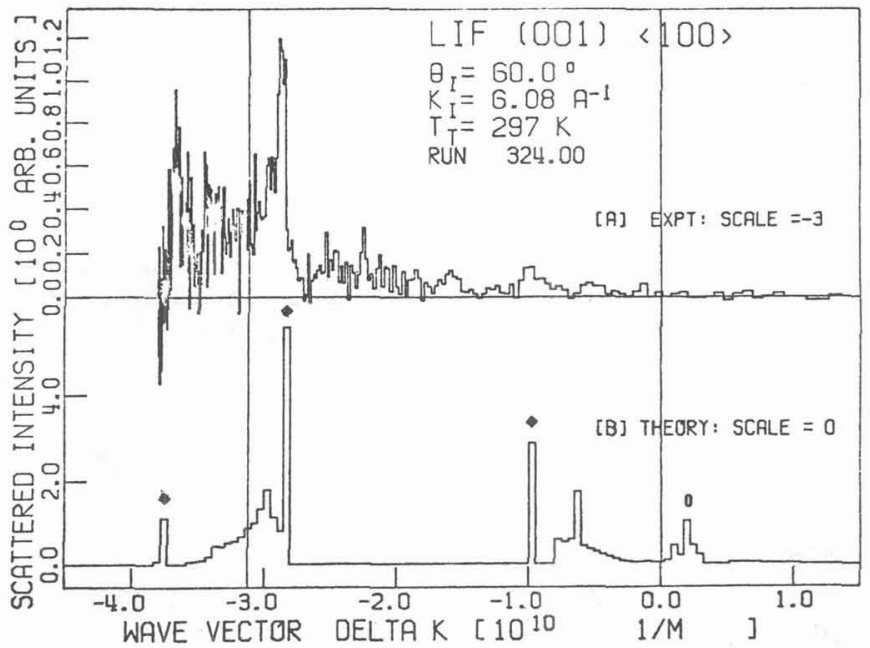

Fig. 5 : Same as fig. 3 for incidence angles $\theta_{i}=60.0^{\circ}$ and $63.2^{\circ}$.

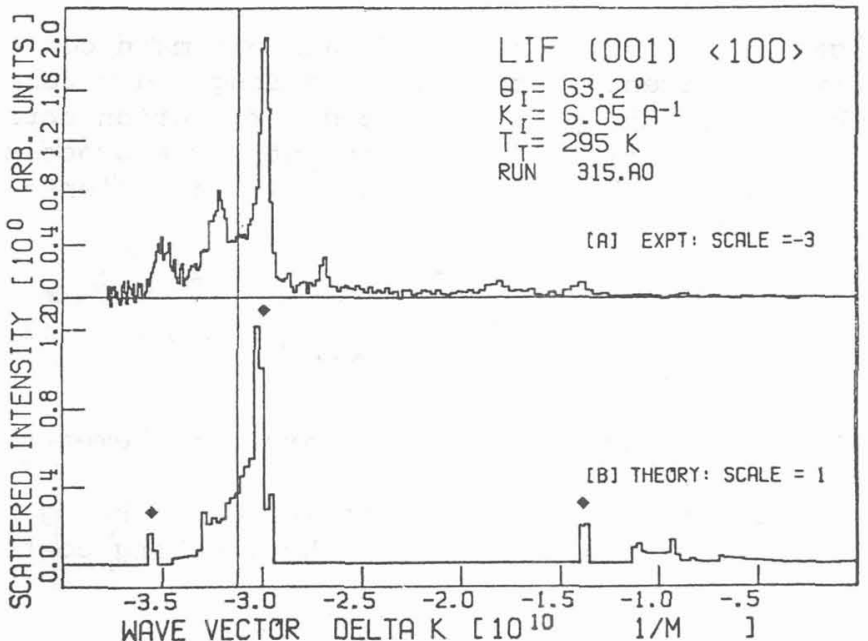

nance. The RW dispersion curve is compared to the atom scattering data (open circles) 25 . The broken line shows the shell model slab calculation by chen et $a 2^{13}$. The quality of bulk dynamics can be judged by comparing to neutron data (black points) the band edges which correspond to bulk dispersion curves along symmetry directions.

In this calculation, based on BSM and room temperature data, bulk $\left(\alpha_{t}\right)$ and surface $\left(\alpha_{ \pm}^{s}\right)$ ion polarizabilities are equal $\left(\alpha_{ \pm}=\alpha_{ \pm}^{s}\right)$ and taken from the classical compilation of Tessmann, Kahn and Shockley 33 (TKS). If $\alpha_{\text {S }}$ is allowed to be larger than $\alpha_{\text {- }}$ and both are adjustable, the residual discrepancies at the $M$ point can be removed in both bulk and RW dispersion curves. ${ }^{29}$ Despite the general argument that surface polarizabilities should be larger than bulk polarizabilities owing to the smaller coordination, we did not use this fitting procedure here, since TKS values yield an excellent fit in NaF and $\mathrm{KCl}$ and a reasonable compromise in LiF.

In evaluating $d^{2} R(1) / d \omega d \Omega$, we use the static surface profile $D_{O}(\vec{R})$ 
which gives the best fit to diffraction amplitudes according to Garcia ${ }^{2}$ The kinematical parameters $\left(k_{i} \simeq 6.1 \mathrm{~A}^{-1} ; \theta_{i}=90^{\circ}-\theta_{f}=49.8^{\circ}, 72.2^{\circ}\right.$, $36.0^{\circ}, 60.0^{\circ}$ and $63.2^{\circ}$ ) correspond to a selected set of ToF spectra measured by Brusdeylins, Doak and Toennies with the apparatus of ref. 25. Experimental and theoretical spectra are shown together in figs 3-5 as functions of $\mathrm{k}$. The phonon energy is readily obtained from eq. 1 with $\mathrm{K}_{\mathrm{p}}=\mathrm{K}$ and $\mathrm{K}_{\mathrm{np}}=0$. The vertical lines correspond to $\mathrm{K}=\mathrm{G}=$ integer $\times 2 \pi / a$ where $a$ is the interionic distance.

The theoretical RW peaks are represented by isolated rectangular peaks (marked by $\bullet$ ). At low energy ( $K$ close to $G$ ) the RW peak is not resolved from the continuous bulk density. Close to $\mathrm{KF}$ condition the RW peak may extend over several bins. This is seen in fig. $3\left(\theta_{i}=49.8^{\circ}\right)$, where the $\mathrm{KF}$ induced broadening of the RW peak $\mathrm{K}$ reflects fairly well the experimental structure. Here the RW peak at $K \sim-0.2$ is not resolved, but the spectra appear to be in reasonably good agreement.

At $\theta_{1}=72.2^{\circ}$, the single RW peak is well resolved letting one appreciate how important is the bulk phonon contribution forming the long tail above $K=-3.4$.

A richer structure is found for $\theta_{i}=36.0^{\circ}$ (fig. 4). Here the intensities of the three distinct RW peaks are in very good agreement with the predicted intensities. Again the experimental tails aside the RW peaks are seen to correspond to bulk phonon structures. While the band around $\mathrm{K}=0$ contains acoustic phonons, the long tail above $K=2.8$ comes essentially from optical phonons. The small theoretical peak 0 is a Lucas optical surface mode $\left(S_{4}\right.$ in $\left.f i g .2\right)$; this is also predicted for $\theta_{1}=49.8^{\circ}$ and $60.0^{\circ}$ (fig. 5 ) but no evidence is found in ToF spectra.

A similar overall agreement is found also in $\mathrm{fig} .5\left(\theta_{i}=60.0^{\circ}\right.$, $\left.63.2^{\circ}\right)$. However, once we fit the maximum intensities around $k=-3$, we note that the RW intensity at larger (smaller) absolute momentum transfer is weaker (stronger) than the observed one.

The general good agreement between theory and experiment means, for the experimentalist, that one-phonon processes are dominant and, for the theoretician, that the Green's function method for surface dynamics and the HCS model employed in scattering theory work quite well. However, the future interest is on the discrepancies. Why does theory predict too large (small) intensities at small (large) momentum transfers ? Why are the predicted Lucas mode peaks not (yet) seen in the experiments?

Fig. 6 : Surface phonon dispersion curves of $\mathrm{KCl}(\mathrm{OOl})$ along the symmetry directions for sagittal ( 1 ) and parallel (||) polarization.

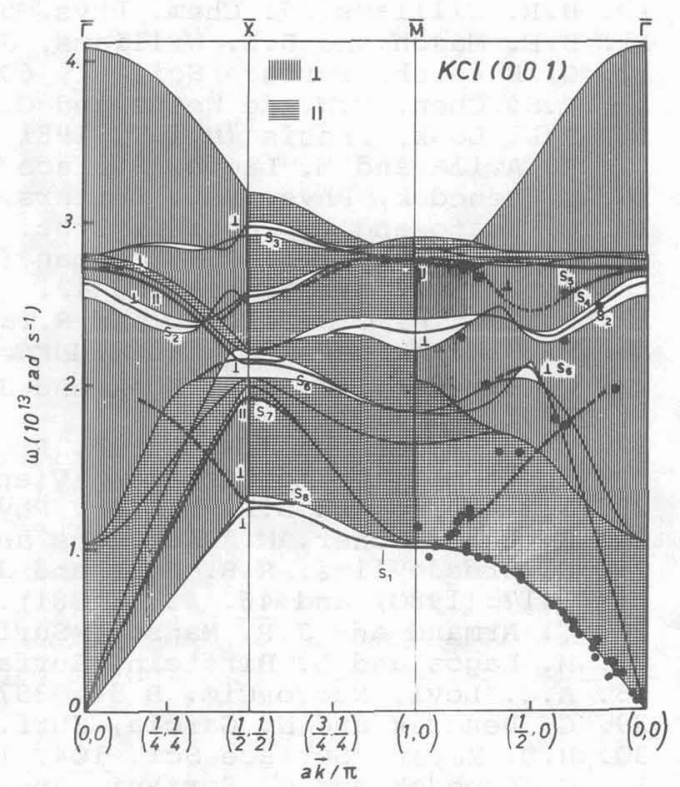


An answer to these questions will probably come from a better description of the surface-atom potential, including the attractive part and the effects of bound-state resonances. For example, the weakness of Lucas modes in scattering could mean that $\mathrm{Li}^{+}$- He interaction is even weaker than that schematically described by the choosen surface profile. Actually, the difficulty existing in LiF for the observation of optical modes is that the radius and the polarizability of L 1 lon (the ion which moves in optical modes) are too small and consequently so are both repulsive and attractive interactions with He atoms.

A better situation occurs in crystals like $\mathrm{KCl}$, where the ions have approximately the same mass and are both polarizable. Moreover in KCl optical frequencies are smaller than $k_{B} T$ (room temperature) and wi, and can therefore be observed in both energy loss and gain processes. Indeed in $\mathrm{KCl}(\mathrm{OOl})$, besides sharp RW peaks, additional structures corresponding to the strong resonance $S_{8}$ are observed in ToF spectra: ${ }^{14}$ such experimental points are compared to the calculated dispersion curves in fig. 6. Further weak and barely resolved features can be related to optical modes of higher frequency $\left(S_{2}, S_{6}\right.$ and $\left.S_{4}\right)$, but such interpretation is still sub judice. We hope that future measurements will confirm this stimulating observation.

\section{References}

1. G. Benedek, Proc: Int. School of Physics "E. Fermi", Course LVIII, ed. F.O. Goodman (Compositori, Bologna 1974), p. 605.

2. K.H. Rieder, Surface Sc1. 26, 637 (1971).

3. J. Sandercock, Solid State Comm. 26, 547 (1978).

4. A. Otto, Z. Physik 216, 398 (1968).

5. H. Ibach, Phys. Rev. Letters 24, 1416 (1970).

6. T. Wolfram, Inelastic Electron Tunnel Spectroscopy (Springer V.1978)

7. N. Cabrera, V.Celli and R.ilanson, Phys.Rev.letters 22, 346 (1969).

8. J.P. Toennies and K. Winckelmann, J. Chem. Phys. 66, 3965 (1977).

9. R.B. Subbarao and D.R. Miller, J. Chem. Phys. 51, 4679 (1969).

10. B.R. Williams, J. Chem. Phys. 55, 1315 and $322 \bar{\delta}(1971)$.

11. B.F. Mason and B.R. Williams, J. Chem. Phys. 61, 1435 (1974).

12. G. Benedek, Surface Sci. 6I, 603 (1976).

13. T.S. Chen, F.W. de Wette and G.P.Alldredge, Phys.Rev. B15,1167 (1977)

14. R.B. Doak, Thesis (M.I.T. 1981, unpublished) and private comm.

15. R. Avila and M. Lagos, Surface Sci. 103, L104 (1981).

16. G. Benedek, Phys. Rev. Letters, $35, \frac{13}{234}$ (1975).

17. G. Boato and P. Cantini, Proc. Int. School of Physics "E.Fermi", Course LVIII, ed. F.O.Goodman (Compositori, Bologna 1974), p. 707.

18. Ref. 13, footnotes 41 and 42 .

19. P. Cantini, G.P.Felcher and R.Tatarek, Phys.Rev.Letters 37,606 (1979)

20. P. Cantini and R. Tatarek, Phys. Rev. B 23, 3030 (1981).

21. G. Brusdeylins, R.B. Doak and J.P. Toennies, J.Chem.Phys. 75 (A), $1781(1981)$.

22. D.R. Miller and J.M. Horne, Proc. 7 th Intern. Vac. Congr. and $3 r d$ Int. Conf. Solid Surfaces (Vienna 1977) p. 1385.

23. J.M. Horne and D.R. Miller, Phys. Rev. Letters 41, 511 (1978).

24. B. Feuerbacher, M.A.Adriaens and H.Thuis, Surf. Sci. 94,1171 (1980).

25. G. Brusdeylins, R.B. Doak and J.P. Toennies, Phys. Rev. Letters 44 , 1417 (1980) and 46,437 (1981).

26. G. Armand and J. $\vec{R}$. Manson, Surface Sci. 80, 532 (1979).

27. M. Lagos and L. Birstein, Surface Sci. $5 \overline{1}, 469$ (1975).

28. A.C. Levi, Nuovo Cim. B 54, 357 (1979).

29. G. Benedek and N. Garcia, Surf.Sci. 80,543(1979); 103, L143 (1981).

30. H.D. Meyer, Surface Sci. 104,117 (1981).

31. G. Benedek and G. Seriani, Jpn. J. Appl. Phys. Suppl.2,pt. 2, 545(1974)

32. N. Garcia and N. Cabrera, Phys. Rev. B18, 576 (1978):

33. J.R. Tessman, A.H. Kahn and W. Shockley, Phys. Rev. 92,890 (1953). 